\title{
ISOMETRIES ON CONSERVATIVE SUBALGEBRAS OF BOUNDED SEQUENCES
}

\author{
J. CONNOR AND I. LOOMIS
}

(Communicated by John B. Conway)

\begin{abstract}
Using extreme point techniques, we show that if $A$ is a closed subalgebra of the bounded sequences which contain $c$, then any linear isometry of $A$ onto itself is a permutation up to a modulus one multiplication. If the subalgebra $A$ is generated by an ideal, then a permutation $P$ maps $A$ onto itself if and only if $P$ maps $\mu$-null sets to $\mu$-null sets where $\mu$ is a $\{0,1\}$ valued finitely additive measure associated with the ideal. In particular, if $T$ is a nonnegative regular summability method, we characterize the isometries which map the bounded strongly $T$-summable sequences onto themselves and give a concrete sufficient condition for a permutation to map the bounded strongly Cesaro summable sequences onto themselves.
\end{abstract}

In this paper we establish that any surjective linear isometry of a closed subalgebra of $\ell_{\infty}$ which contains the convergent sequences can be realized as modulus one multiplication of a permutation and characterize the linear isometries for a subclass of these algebras. We also give an example of a closed subalgebra that has isometries which cannot be realized as permutations.

Banach [1] was the first to determine that the surjective linear isometries of the spaces $\ell_{p}, 1 \leq p<\infty(p \neq 2)$, were modulus one multiples of permutation maps. Since then, various authors have studied the isometries of sequence spaces possessing certain properties. For example, see [5],[6],[10],[11], and [13]. It is not surprising that the isometries found in these papers are modulus one multiples of permutations and results of Tam [11], Fleming and Jamison [6], and [7], Fleming, Goldstein, and Jamison [5] and Zaidenberg [13] allow one to deduce that the surjective linear isometries of $\ell_{\infty}$ are of this form. What is surprising is that it appears that no one has specifically addressed the space $\ell_{\infty}$ and its subalgebras, including $c$.

Received by the editors March 3, 1989.

1980 Mathematics Subject Classification (1985 Revision). Primary 47B37; Secondary 46B25.

Key words and phrases. Linear isometry, subalgebras of bounded sequences, strong matrix summability. 
In the following, let

$$
\begin{aligned}
\omega & =\text { the collection of all complex valued sequences } \\
\varphi & =\{x \in \omega: x \text { is finitely nonzero }\} \\
c_{0} & =\{x \in \omega: \lim x=0\} \\
c & =\{x \in \omega: \lim x \text { exists }\} \\
\ell_{\infty} & =\left\{x \in \omega: \sup _{n}\left|x_{n}\right|<\infty\right\}
\end{aligned}
$$

and let $\|x\|=\sup _{n}\left|x_{n}\right|$ whenever $x \in \ell_{\infty}$. We let $e$ denote the sequence that is identically $1, e^{k}$ denote the sequence with 1 in the $k$ th position and 0 elsewhere and, for $x \in \omega$, we write $|x|=1$ if $\left|x_{k}\right|=1$ for all $k \in \mathbf{N}$. If $\mathscr{A}$ is a subalgebra of $\ell_{\infty}$, we say that $\mathscr{A}$ is conservative if $c \subseteq \mathscr{A}$. Note that a conservative subalgebra always contains the identity. A linear mapping $T$ from $\mathscr{A}$ onto itself is called an isometry if $\|T x\|=\|x\|$ for all $x \in \mathscr{A}$. Given $k \in \mathbf{N}$, we let $p_{k}(x)=x_{k}$ for all $x \in \ell_{\infty}$ and note that $p_{k} \in \mathscr{A}^{*}$ (the continuous dual of $\mathscr{A}$ ).

We also use some of the language of general matrix summability theory. If $S=\left(s_{n, k}\right)$ is an infinite array of scalars where $k$ and $n$ range over $\mathbf{N}$ and $x, y \in \omega$, we say that $S x=y$ if $\sum_{k=1}^{\infty} s_{n, k} x_{k}=y_{n}$ for all $n \in \mathbf{N}$ and call $S$ a matrix summability method. The matrix $S$ is called nonnegative if $s_{n, k} \geq 0$ for all $n, k \in \mathbf{N}$. A matrix summability method is called regular if $S x \in c$ and $\lim S x=\lim x$ whenever $x \in c$ and a sequence is said to be strongly $S$-summable to $L$ if $\lim _{n} \sum_{k=1}^{\infty} s_{n, k}\left|x_{k}-L\right|=0$.

\section{ISOMETRIES ON CONSERVATIVE SUBALGEBRAS OF $\ell_{\infty}$}

The main result of this section is that if $\mathscr{A}$ is a closed conservative subalgebra of $\ell_{\infty}$ and $T$ is a linear isometry of $\mathscr{A}$ onto itself, then $T$ is a permutation up to signs, i.e. $T x=\left(\sigma_{k} x_{\pi(k)}\right)$ where $\pi: \mathbf{N} \rightarrow \mathbf{N}$ is permutation and $\left|\sigma_{k}\right|=1$ for all $k \in \mathbf{N}$. We establish this by exploiting the observations that if $T$ is an isometry, then $T^{*}$ is an isometry and that isometries carry extreme points to extreme points.

We begin by observing that if $f$ is an extreme point of $B_{\mathscr{A}^{*}}$, the ball of $\mathscr{A}^{*}$, then there is a multiplicative linear functional $m \in \mathscr{A}^{*}$ and an $\alpha \in \mathbf{C},|\alpha|=1$, such that $f(x)=\alpha m(x)$ for all $x \in \mathscr{A}$. Recall that if

$$
K=\left\{m \in B_{\mathscr{W}}: m(x y)=m(x) m(y) \text { and } m(\bar{x})=m \overline{(x)} ; x, y \in \mathscr{A}\right\}
$$

then $K$ is a weak-star compact subset of $\mathscr{A}^{*}$ and $R: \mathscr{A} \rightarrow C(K)$ defined by $(R x)(m)=m(x)$ is a surjective linear isometry. The Arens-Kelly theorem asserts that if $z^{*}$ is an extreme point of $B_{C(K)^{*}}$, then there is an $m \in K$ and an $\alpha \in \mathbf{C}$ such that $z^{*}=\alpha x_{m}^{*}$ where $|\alpha|=1$ and $x_{m}^{*}(f)=f(m)$ for all $f \in C(K)$. Since $\left(R^{*} x_{m}^{*}\right)(x)=m(x)$ for all $x \in \mathscr{A}$, isometries carry extreme points to extreme points and $R^{*}$ and $\left(R^{*}\right)^{-1}$ are both isometries, we may conclude that 
any extreme point of $B_{\mathscr{A}}$. is of the form $\alpha m$ where $|\alpha|=1$ and $m \in K[4$, IV.6.18].

Theorem 1. Let $\mathscr{A}$ be a closed subalgebra of $\ell_{\infty}$ which contains $c$ and $T: \mathscr{A} \rightarrow$ $\mathscr{A}$ be a surjective linear isometry. Then there exists a permutation $\pi: \mathbf{N} \rightarrow \mathbf{N}$ and a sequence $\left(\sigma_{k}\right) \in \mathscr{A},|\sigma|=1$, such that $T x=\left(\sigma_{k} x_{\pi(k)}\right)$ for all $x \in \mathscr{A}$; i.e., $T$ is a permutation up to signs.

Proof. Let $k \in \mathbf{N}$ and define $f_{k} \in \mathscr{A}^{*}$ by $f_{k}(x)=(T x)_{k}$ for all $x \in \mathscr{A}$. Note that $\left\|f_{k}\right\|=1$ and $f_{k}=T^{*} p_{k}$ for all $k \in \mathbf{N}$. Since $p_{k}$ is an extreme point of $B_{\mathscr{O}^{*}}$ and $T^{*}$ is an isometry, $f_{k}$ is also an extreme point of $B_{\mathscr{A}^{*}}$ and hence $f_{k}=\sigma_{k} m_{k}$ where $\left|\sigma_{k}\right|=1$ and $m_{k}$ is a multiplicative linear functional on $\mathscr{A}$.

Now set $I_{r}=\left\{k \in \mathbf{N}: m_{k}\left(e^{r}\right) \neq 0\right\}$ for all $r \in \mathbf{N}$. Since $\left\|T e^{r}\right\|=1$, there is a $k \in \mathbf{N}$ such that $\left(T e^{r}\right)_{k} \neq 0$ and hence $m_{k}\left(e^{r}\right) \neq 0$, i.e. $I_{r} \neq \varnothing$ for all $r \in \mathbf{N}$. Also note that if $r \neq s$, then $m_{k}\left(e^{r} e^{s}\right)=m_{k}(0)=0$ and hence $I_{r} \cap I_{s}=\varnothing$. It follows that the mapping $\beta: \mathbf{N} \rightarrow \mathbf{N}$ defined by $\beta(r)=\min \left\{k: m_{k}\left(e^{r}\right) \neq 0\right\}$ is well defined and injective.

Before establishing that $\beta$ is also surjective, we show that if $z \in c_{0}$ then $m_{\beta(r)}(z)=z_{r}$. Since $m_{\beta(r)}\left(e^{r}\right)^{2}$ and $m_{\beta(r)}\left(e^{r}\right) \neq 0$, we have that $m_{\beta(r)}\left(e^{r}\right)=1$ and $m_{\beta(r)}\left(e^{s}\right)=0$ whenever $s \neq r$. It follows then if $z \in \varphi$, then $m_{\beta(r)}(z)=$ $\sum_{j=1}^{\infty} z_{j} m_{\beta(r)}\left(e^{j}\right)=z_{r} m_{\beta(r)}\left(e^{r}\right)=z_{r}$. Since $\bar{\varphi}=c_{0}, m_{\beta(r)}(z)=z_{r}$ for all $z \in c_{0}$.

Next we establish that $m_{\beta(r)}(x)=x_{r}$ for all $x \in \mathscr{A}$. Let $r \in \mathbf{N}$ be fixed and let $x \in \mathscr{A}, x \notin c_{0}$. Let $\tilde{x}=x-x_{r} e^{r}, z=\tilde{x} /\|\tilde{x}\|$ and $\alpha=m_{\beta(r)}(z)$. Note that $\left|m_{\beta(r)}\left(z \pm e^{r}\right)\right|=|\alpha \pm 1| \leq\left\|z \pm e^{r} \mid\right\|\left\|m_{\beta(r)}\right\|=1$ forces $\alpha=0$. It follows that $m_{\beta(r)}(x)=m_{\beta(r)}\left(x_{r} e^{r}+\tilde{x}\right)=x_{r}$ and hence we have that $m_{\beta(r)}(x)=x_{r}$ for all $x \in \mathscr{A}$.

We are now prepared to show that $\beta$ is surjective. Suppose that $k \in$ $\mathbf{N} \backslash\{\beta(r): r \in \mathbf{N}\}$. Since $T$ is surjective, there is an $x \in \mathscr{A}$ such that $T x=e^{k}$. Since $(T x)_{\beta(r)}=\sigma_{r} m_{\beta(r)}(x)=\sigma_{r} x_{r}$ for all $r \in \mathbf{N}$, we have that $x=0$ and consequently $T x=e^{k}=0$. It follows that $\mathbf{N}=\{\beta(r): r \in \mathbf{N}\}$. Also note that $T e=\sigma$ forces $\sigma \in \mathscr{A}$.

Define the permutation $\pi$ of $\mathbf{N}$ by $\pi=\beta^{-1}$. Now $T x=\left(\sigma_{k} m_{k}(x)\right)=$ $\left(\sigma_{k} m_{\beta(\pi(k))}(x)\right)=\left(\sigma_{k} x_{\pi(k)}\right)$ for all $x \in \mathscr{A}$.

The preceding proof makes extensive use of the hypothesis that $c \subseteq \mathscr{A}$ in that we exploit that $\bar{\varphi}=c_{0} \subseteq \mathscr{A}$ and $e \in \mathscr{A}$. There is, however, a large collection of closed subalgebras of $\ell_{\infty}$ that do not contain $c$. In fact, if $K$ is an infinite compact metric space which is dense in itself then there is a closed subalgebra $U$ of $\ell_{\infty}$ such that $U$ is isometrically isomorphic to $C(K)$ and $U$ does not contain $c$ : If $\left(x_{n}\right)$ is a dense sequence of distinct elements of $K$ then $U$ can be taken to be the image of $C(K)$ under $S$ where $S f=\left(f\left(x_{n}\right)\right)$ for all $f \in C(K)$. Note that if $\left(f\left(x_{n}\right)\right)$ is convergent then $f$ is constant; hence $U$ cannot contain $c$. 
The above collection of examples indicates that the hypothesis $c \subseteq \mathscr{A}$ cannot be completely removed from the theorem. In fact, let $r=\left(r_{n}\right)$ be an enumeration of the rational numbers in $[0,1]$ and let $R$ be the linear isometry on $C[0,1]$ defined by $R f(x)=f(\sqrt{x})$. Define $S: C[0,1] \rightarrow \ell_{\infty}$ by $S f=\left(f\left(r_{n}\right)\right)$. If $\mathscr{A}$ is the image of $C[0,1]$ under $S$ and $T: \mathscr{A} \rightarrow \mathscr{A}$ is defined by $T=S R S^{-1}$, then $T$ is an isometry of $\mathscr{A}$ onto itself and $\operatorname{Tr}=\left(\sqrt{r}_{n}\right)$. Since $\left(\sqrt{r}_{n}\right)$ contains an irrational number, it follows that $T$ cannot be represented as a permutation up to a modulus one multiplication.

\section{ISOMETRIES ON SUBALGEBRAS GENERATED BY IDEALS WHICH CONTAIN $c_{0}$}

In the preceding section we established that any isometry of a conservative subalgebra onto itself can be realized as a permutation. The converse, however, does not hold for arbitrary conservative subalgebras. In order to see this, recall that if $x$ and $y$ are both divergent sequences of 0 's and 1's then there is a permutation $\pi$ such that $y_{k}=\left(x_{\pi(k)}\right)$. It follows immediately that if $\mathscr{A}$ is a subalgebra such that there are divergent sequences of 0 's and 1's such that $x \in \mathscr{A}$ but $y \notin \mathscr{A}$, then there is a permutation $P$ that does not map $\mathscr{A}$ onto itself. We will give an example of a collection of such conservative subalgebras later in this section.

In this section we characterize the isometries on proper subalgebras "generated by ideals which contain $c_{0}$ " which are constructed as follows: Let $M$ be a proper closed ideal of $\ell_{\infty}$ such that $c_{0} \subseteq M$ and set

$$
\mathscr{A}_{M}=\left\{x \in \ell_{\infty}: x-L e \in m \text { for some } L \in \mathbf{C}\right\} .
$$

It is easy to verify that $\mathscr{A}_{M}$ is a closed subalgebra of $\ell_{\infty}$, and since $c_{0} \subseteq$ $M, c \subseteq \mathscr{A}_{M}$. Also, note that if $x-L e \in M$ then $x$ has a subsequence which is convergent to $L$ (otherwise $M=\ell_{\infty}$ ). Throughout the sequel, we always let $M$ denote a closed ideal of $\ell_{\infty}$ such that $c_{0} \subseteq M$ and, if $x \in \ell_{\infty}$ and $\varepsilon>0, A(\varepsilon, x)=\left\{k \in \mathbf{N}:\left|x_{k}\right| \geq \varepsilon\right\}$. If $L \in \mathbf{C}$ we let $x-L$ denote the sequence $x-L e$.

It is convenient to describe subalgebras using finitely additive two-valued measures. Given $M$, set $\Gamma_{M}=\left\{A \subseteq N ; \chi_{A}\right.$ or $\left.\chi_{A^{c}} \in M\right\}$ and define $\mu_{m}: \Gamma_{M} \rightarrow$ $\{0,1\}$ by $\mu_{M}(A)=0$ if $\chi_{A} \in M$ and $\mu_{M}(A)=1$ if $\chi_{A^{c}} \in M$. It is straightforward to check that $\Gamma_{M}$ is a subfield of $\mathbf{P}(\mathbf{N})$, the power set of $\mathbf{N}$, and that $\mu_{M}$ is a finitely additive measure. Also note that if $B \in \Gamma_{M}, \mu_{M}(B)=0$ and $A \subseteq B$, then $\mu_{M}(A)=0$; i.e., $\mu_{M}$ is complete [3].

The following lemma provides a useful test for membership in a closed ideal of $\ell_{\infty}$.

Lemma 2. Let $x \in \ell_{\infty}$ and $M$ be a closed ideal of $\ell_{\infty}$. Then $x \in M$ if and only if $\mu_{M}(A(\varepsilon, x))=0$ for all $\varepsilon>0$.

Proof. Observe that $\chi_{A}(\varepsilon, x)=x y$ if $y \in \ell_{\infty}$ is defined by $y_{k}=1 / x_{k}$ when $\left|x_{k}\right| \geq \varepsilon$ and 0 otherwise. Since $M$ is an ideal, it follows that if $x \in M$ then $\chi_{A(\varepsilon, x)} \in M$ for all $\varepsilon>0$. 
Now note that $\left\|x-x \chi_{A(\varepsilon, x)}\right\|<\varepsilon$ for all $\varepsilon>0$. Since $M$ is closed, we have that $x \in M$ if $\chi_{A(\varepsilon, x)} \in M$ for all $\varepsilon>0$.

A consequence of this lemma is that $x \in \mathscr{A}_{M}$ if and only if

$$
\mu_{M}(A(\varepsilon, x-L))=0
$$

for all $\varepsilon>0$ for some $L \in \mathbf{C}$.

There is an abundant collection of such subalgebras. If $S=\left(s_{n, k}\right)$ is a nonnegative regular summability method, then

$$
M(S)=\left\{x \in \ell_{\infty} \lim _{n}: \sum_{k=1}^{\infty} s_{n, k}\left|x_{k}\right|=0\right\}
$$

is a closed ideal of $\ell_{\infty}$ [3], [8] and the subalgebra $\mathscr{A}_{M(S)}$ consists of the bounded strongly $S$-summable sequence. Since $S$ is regular, Steinhaus's theorem on summability [12] yields that there is a sequence of 0's and 1's that is not strongly $S$-summable and hence $\mathscr{A}_{M(S)} \neq \ell_{\infty}$. We also note that

$$
\Gamma_{M(S)}=\left\{A \subset \mathbf{N}: \lim _{n} \sum_{k=1}^{\infty} s_{n, k} \chi_{A}(k)=0 \text { or } 1\right\}
$$

and $\mu_{M(S)}(A)=\lim _{n} \sum_{k=1}^{\infty} s_{n, k} \chi_{A}(k)$ for all $A \in \Gamma_{M(S)}$. It can also be shown that $\mathscr{A}_{M(S)}$ is the collection of bounded strongly $S$-summable sequences [3].

Theorem 3. Let $\mathscr{A}_{M}$ be the subalgebra generated by $M$ and suppose $\mathscr{A}_{M} \neq \ell_{\infty}$. Then $T$ is a linear isometry $\mathscr{A}_{M}$ onto itself if and only if $T x=\left(\sigma_{k} x_{\pi(k)}\right)$ where

(1) $\left(\sigma_{k}\right) \in \mathscr{A}_{M}$ and $\left|\sigma_{k}\right|=1$ for all $k \in \mathbf{N}$;

(2) $\pi$ is a permutation of $\mathbf{N}$ such that $\pi(A) \in \Gamma_{M}$ if and only if $A \in \Gamma_{M}$;

(3) $\mu_{M}(A)=0$ if and only if $\mu_{M}(\pi(A))=0$ for all $A \in \Gamma_{M}$.

Proof. We let $P x=\left(x_{\pi(k)}\right)$ and $M_{\sigma}(x)=\left(\sigma_{k} x_{k}\right)$ for all $x \in \ell_{\infty}$, and we let $\mu=\mu_{M}, \mathscr{A}=\mathscr{A}_{M}$ and $\Gamma=\Gamma_{M}$.

Sufficiency. We establish that $M_{\sigma}$ and $P$ are both linear isometries of $\mathscr{A}$ onto itself and hence $T=M_{\sigma} P$ is a surjective linear isometry.

First note that, since $\left|\sigma_{k}\right|=1$ for all $k,\left\|M_{\sigma} x\right\|=\|x\|$ for all $x \in \mathscr{A}$ and the sequence $\sigma^{-1}=\left(\sigma_{k}^{-1}\right) \in \ell_{\infty}$. Since $\sigma \in \mathscr{A}, M_{\sigma} x \in \mathscr{A}$ for all $x \in \mathscr{A}$ and there is an $L \in \mathbf{C}$ such that $\sigma-L \in M$. Since some subsequence of $\sigma$ must converge to $L,|L|=1$ and $L^{-1}$ exists. Now $-L^{-1} \sigma^{-1}(\sigma-L e)=\sigma^{-1}-L^{-1} \in M$ and hence $\sigma^{-1} \in \mathscr{A}$. It follows immediately that $M_{\sigma}$ is an isometry of $\mathscr{A}$ onto itself.

Next we establish that $P$ is a linear isometry of $\mathscr{A}$ onto itself. First we establish that $P$ maps $M$ into $M$. Let $x \in M$ and observe that

$$
\begin{aligned}
A(\varepsilon, P x) & =\left\{r:\left|(P x)_{r}\right| \geq \varepsilon\right\}=\left\{r:\left|x_{\pi(r)}\right| \geq \varepsilon\right\} \\
& =\left\{\pi^{-1}(s) ;\left|x_{s}\right| \geq \varepsilon\right\}=\pi^{-1}(A(\varepsilon, x)) .
\end{aligned}
$$


Since $\mu(A(\varepsilon, x))=0, \mu\left(\pi^{-1}(A(\varepsilon, x))=\mu(A(\varepsilon, P x))=0\right.$ for all $\varepsilon>0$ and hence $P x \in M$. If $y \in \mathscr{A}$, then $y-L e \in M$ for some $L$ and hence $P(y-L e)=P y-L e \in M$, i.e., $P \mu \in \mathscr{A}$.

Since the same argument shows that $P^{-1}$ maps $\mathscr{A}$ into $\mathscr{A}$ and it is clear that $P$ is a linear isometry, we have that $P$ is a surjective linear isometry.

Necessity. Now suppose that $T: \mathscr{A} \rightarrow \mathscr{A}$ is a surjective linear isometry. We have already established that there is a permutation $\pi$ and $\sigma \in \mathscr{A},\left|\sigma_{k}\right|=1$ for all $k$, such that $T=M_{\sigma} P$. The proof will be completed once we have established (2) and (3).

The preceding part of the proof establishes that $\sigma^{-1} \in \mathscr{A}$. It follows that $P=M_{\sigma^{-1}} M_{\sigma} P$, and hence $P^{-1}$, are linear isometries of $\mathscr{A}$ onto itself. Also, if $A \in \Gamma$ then $P\left(\chi_{A}\right)$ and $P^{-1}\left(\chi_{A}\right)$ are both in $\mathscr{A}$. Since $P^{-1}\left(\chi_{A}\right)=\chi_{\pi(A)}$, and hence either $\chi_{\pi(A)}$ or $e-\chi_{\pi(A)}=\chi_{\pi(A)^{c}}$ must be in $M$, we have that $\pi(A) \in \Gamma$ if $A \in \Gamma$. Similarly, since $P\left(\chi_{A}\right)=\chi_{\pi^{-1}(A)}$, we have that $A \in \Gamma$ if $\pi(A) \in \Gamma$.

Now suppose that $A \in \Gamma, \mu(A)=0$ and $\mu(\pi(A))=1$. Since $\mathscr{A} \neq \ell_{\infty}$, there is a $D \subseteq \mathbf{N}$ such that $\chi_{D} \notin \mathscr{A}$ [2]. Write $A \cup D=A \cup F$ where $A \cap F=\varnothing$ and note that $F \notin \Gamma$. Since $\pi(F) \subseteq \pi(A)^{c}$ and $\mu\left(\pi(A)^{c}=0\right.$, the completeness of $\mu$ forces $F \in \Gamma$. The necessity of (3) follows and hence the theorem.

As the next example indicates, the hypothesis $\mathscr{A}_{M} \neq \ell_{\infty}$ cannot be eliminated from the preceding theorem. Mazur [9] has established the existence of a linear functional $f \in \ell_{\infty}^{*}$ such that $f(x y)=f(x) f(y)$ for all $x, y \in \ell_{\infty}$ and $f(x)=$ $\lim x$ for all $x \in c$. Observe that, for any $A \subseteq \mathbf{N}, f\left(\chi_{A}\right)=f\left(\chi_{A}\right)^{2}$ and hence $f\left(\chi_{A}\right)=0$ or $f\left(\chi_{A}\right)=1$. If we set $M=\left\{x \in \ell_{\infty}: f(x)=0\right\}, M$ is a closed ideal, $\Gamma_{M}=P(\mathbf{N})$ and $\mu_{M}(A)=f\left(\chi_{A}\right)$. Also note that if $x \in \ell_{\infty}$ and $f(x)=L$, then $x-L \in M$ and hence $\mathscr{A}_{M}=\ell_{\infty}$. Now let $A \subseteq \mathbf{N}$ such that $\chi_{A} \notin c$ and $\pi$ be a permutation such that $\pi(A)=A^{c}$. Since any permutation determines an isometry of $\ell_{\infty}$ onto itself, $\pi$, determines an isometry of $\mathscr{A}_{M}$ onto itself that does not take $\mu_{M}$-null sets to $\mu_{M}$-null sets.

We can use the above theorem to give a sufficient condition for an isometry to map the bounded strongly Cesaro sequences onto themselves. First recall that a sequence $x=\left(x_{k}\right)$ is strongly Cesaro summable to $L$ provided $\lim _{n} n^{-1} \sum_{k=1}^{n}\left|x_{k}-L\right|=0$. It can be established that a bounded sequence is strongly Cesaro summable to $L$ if and only if $\lim _{n} n^{-1} \sum_{k=1}^{n} \chi_{A(\varepsilon, x-L)}(k)=0$ for all $\varepsilon>0$ [3]. Let $S$ denote the collection of bounded sequences which are strongly Cesaro summable to 0 . It is now straightforward to verify that $S$ is an ideal which, via Lemma 2 , is closed in $\ell_{\infty}$. Also note that, by Steinhaus's theorem on summability $A_{S} \neq \ell_{\infty}$.

Definition 4. We call a permutation $\pi$ of $\mathbf{N}$ a block permutation if there is a sequence of integers $1=\nu(1)<\nu(2)<\cdots$ and permutations

$$
\pi_{k}:[\nu(k), \nu(k+1)) \rightarrow[\nu(k), \nu(k+1))
$$

such that $\pi_{k}(r)=\pi(r)$ for all $r \in[\nu(k), \nu(k+1))$. 
Proposition 5. Suppose $\pi$ is a block permutation of $\mathbf{N}$ as described in the preceding definition. If $\lim \sup _{k} \nu(k+1) / \nu(k)=1$, then the mapping $P x=\left(x_{\pi(k)}\right)$ maps the bounded strongly Cesaro summable sequences onto themselves.

Proof. A straightforward computation shows that $\chi_{A} \in S$ if and only if $\chi_{A} \in S$ which, by the preceding theorem, establishes the proposition.

\section{REFERENCES}

1. S. Banach, Theorie des operations lineaires, Monografie Mat., PWN Warsaw, 1932.

2. G. Bennett, and N. J. Kalton, Inclusion theorems for $K$-Spaces, Canad. J. Math, 25, No. 3 , 1973, 511-524.

3. J. Connor, Two valued measures and summability, preprint, 1988.

4. N. Dunford and J. Schwartz, Linear operators, part I, Interscience Publishers, New York, 1958.

5. R. Fleming, J. Goldstein and J. Jamison, One-parameter groups of isometries on certain Banach spaces, Pacific J. Math. 64, No. 1 (1976), 145-151.

6. R. Fleming and J. Jamison, Isometries of certain Banach spaces, J. London Math. Soc. 9, No. 2 (1974), 121-127.

7. __ Hermitian operators and isometries on sums of Banach spaces, preprint.

8. A. Freedman and J. Sember, Densities and summability, Pacific J. Math 95, No. 2 (1981), 293-305.

9. S. Mazur, On the generalized limit of a bounded sequence, Colloq. Math. 2 (1951), 173-175.

10. O. I. Skorik, On the isometries of one class of ideals of coordinate spaces, Theory of Functions, Functional Analysis and Its Applications, 120-131 (In Russian).

11. K. W. Tam, Isometries of certain functional spaces, Pacific J. Math. 31, no. 1 (1969), 233-246.

12. A. Wilansky, Summability through functional analysis, North-Holland, Amsterdam, 1984.

13. M. G. Zaidenberg, On isometric classification of symmetric spaces, Soviet Math. Dokl. 18, No. 3 (1977), 636-640.

Department of Mathematics, Ohio University, Athens, Ohio 45701

Department of Mathematics, University of Tennessee at Chattanooga, chattanooga, TENNESSEE, 37403 